\title{
Psychiatric Disorders of Neurocysticercosis: Narrative Review
}

\author{
Asmaa M El-Kady (D) \\ Khaled S Allemailem (iD ${ }^{2}$ \\ Ahmad Almatroudi (iD) ${ }^{2}$ \\ Birgit Abler ${ }^{3}$ \\ Mohamed Elsayed ${ }^{3}$ \\ 'Department of Medical Parasitology, \\ Faculty of Medicine, South Valley \\ University, Qena, Egypt; ${ }^{2}$ Department of \\ Medical Laboratories, College of Applied \\ Medical Sciences, Qassim University, \\ Buraydah, Saudi Arabia; ${ }^{3}$ Department of \\ Psychiatry and Psychotherapy III, \\ University of Ulm, Ulm, 89075, Germany
}

Video abstract

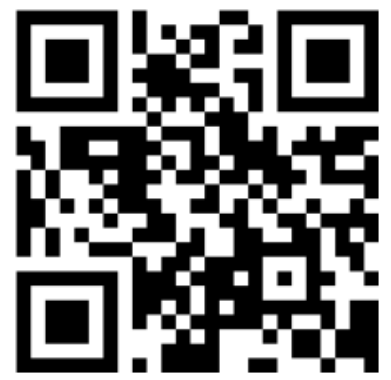

Point your SmartPhone at the code above. If you have a $Q R$ code reader the video abstract will appear. Or use: https://youtu.be/-HGXuA3fOWI
Correspondence: Asmaa M El-Kady

Department of Medical Parasitology,

Faculty of Medicine, South Valley

University, Qena, Egypt

Tel +20 I I I422974 I

Email Asmaa.elkady@med.svu.edu.eg

\begin{abstract}
Neurocysticercosis, the most common type of neuroparasitosis, is a condition in which the central nervous system (CNS) is infested with the pork tapeworm Taenia solium cysticercosis' larvae. Neurocysticercosis is the most widespread parasitic CNS disease worldwide, affecting more than 50 million individuals. As neurocysticercosis is prevalent in developing countries, the growing number of migrants and travelers increases prevalence in developed countries. Possible neuropsychiatric manifestations are depression, cognitive dysfunction, dementia, and visual hallucinations. Depending on the cysts' location in the CNS, focal neurology or psychiatric symptoms manifest. The diagnosis of neurocysticercosis is based on neuroimaging and serology. The correlation between specific symptoms and the cyst's location might help better understand psychiatric disorders' pathophysiology. Nonetheless, the exact prevalence of neurocysticercosis is seldom reported in patients with psychiatric disorders, which may be due to the lack of imaging availability in developing countries with a high prevalence.
\end{abstract}

Keywords: psychiatric, neurocysticercosis, cognitive functions, depression, dementia

\section{Introduction}

Neurocysticercosis is a condition in which the central nervous system (CNS) is infested with the pork tapeworm Taenia solium cysticercosis' larvae. ${ }^{1}$ It is the most common type of neuroparasitosis. ${ }^{1}$ As neurocysticercosis is prevalent in developing countries, the growing number of migrants and travelers increases prevalence in developed countries as well. ${ }^{1}$ Unfortunately, physicians are largely unaware of the epidemiological importance of neurocysticercosis and its psychological issues. Neurocysticercosis is a standard differential diagnosis of most neurological, but not psychiatric, conditions in the endemic regions. However, the infestation results in complex physiological, neurosurgical, and psychological syndromes.

Along with neurobehavioral disorders associated with neurological and neurosurgical problems, the symptoms mimic different psychiatric disorders in up to $15 \%$ of patients with neurocysticercosis. ${ }^{2}$ For example, in the 1960s in England, Dixon and Lipscomb registered psychiatric manifestations in 39 soldiers (affective disorder in 11, schizophrenia in 3 , hysteria in 1 , and psychoneurosis with hysterical features in 2 subjects) among 450 soldiers who acquired neurocysticercosis in service abroad (Proceedings of the Royal Society of Medicine.). It is not precisely clarified when neurocysticercosis was verified for the first time in medical history. Paracelsus wrote his masterwork in 1536, in which he believed that a sick priest's epilepsy resulted from the existence of brain cysts. ${ }^{3}$ We owe Johannes Udalric 
Rumler for the first reference to a case of human cysticercosis in $1558 .^{4,5}$ Domenico Panarolus noted similar cysts in the corpus callosum of a patient's brain who had an epileptic seizure. However, the cysts were not linked to a parasite until 1697, when Marcello Malpighi discovered the zoonotic origin of these cysts and described the scolex, ie, the tapeworm larva within it. ${ }^{6}$ In 1784, Johann August Ephraim Goeze, alien to the Malpighi's work, re-examined the cysticerci, ie, encysted tapeworm larvae in pigs and identified its helminthic nature. Two years later, P.C. Werner rediscovered human cysticercosis in the autopsy of a soldier. ${ }^{6}$ In 1829 , cysticercus infestation of the eye's anterior chamber was observed, representing the first investigation conducted on active cysticercus in a living person. ${ }^{7}$ In 1860, Virchow, who also described this parasite called cysticercus multilocularis, presented the first description of the macropathology and the respective clinical correlations. ${ }^{8}$ The term "cysticerci multilocularis by cysticercus racemose" was permanently changed by Zenker in 1882. Later, Griesinger studied a series of 86 patients in 1862, identified a direct association between cerebral cysticercosis and epilepsy, and proposed a first disease classification. Three years later, Kuchenmeister discovered that the larval stage of the tapeworm Taenia solium is cysticercus. ${ }^{9}$ Between 1909 and 1911, Moses and Wimberg conducted serological experiments using complement fixation in serum for diagnostic purposes and discovered an incidence of neurocysticercosis in specialized hospitals in Mexico of $4 \%{ }^{9}$

\section{Epidemiology Today}

In 2015, T. solium was named by the WHO Foodborne Disease Burden Epidemiology Reference Group as a leading cause of deaths from foodborne illnesses, Figure 1. The parasite was associated with a substantial number of 2.8 million disability-adjusted life-years. ${ }^{10}$ In less developed countries where pigs are raised as a food source close to human dwellings, T. solium infection and the subsequent manifestation of neurocysticercosis are endemic. ${ }^{11,12}$ Neurocysticercosis is the most widespread parasitic CNS disease worldwide, affecting more than 50 million individuals. ${ }^{13,14}$ It is prevalent in Latin America, most of Asia, Sub-Saharan Africa, and parts of Oceania. It is the world's most prominent cause of epilepsy. ${ }^{14}$ Due to the immigration of tapeworm carriers from endemic areas, its diagnosis has significantly increased in more developed countries. ${ }^{1}$ A few populationbased prevalence studies integrating neuroimaging are also

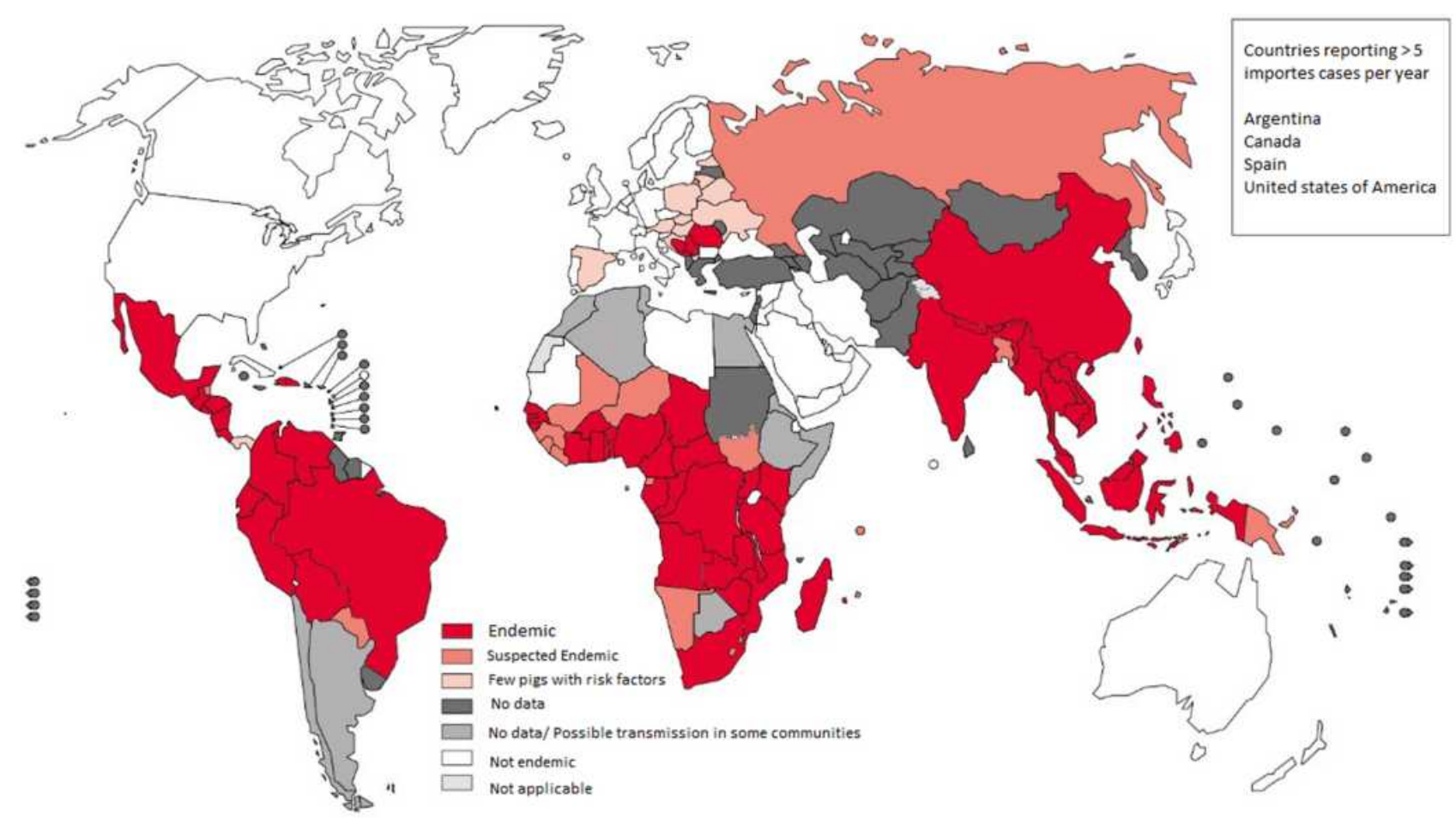

Figure I World map showing the distribution of $T$. solium taeniasis/cysticercosis transmission.

Notes: Reproduced from Garcia HH, Gonzalez AE, Gilman RH. Cysticercosis and Its Impact in Neurological Disease. Clin Microbiol Rev. 2020;33(3):e00085-19. ${ }^{3}$ Copyright (c) 2020 American Society for Microbiology. Reproduced with permission from the American Society for Microbiology. 
available. Epidemiological studies using brain CT (or more rarely using MRI) revealed that 10 to $20 \%$ of villagers in Tanzania have cerebral cysticercosis, most of them with only one or a few calcified scars and no clinical symptoms. ${ }^{10,15}$ In Mexico's endemic villages, approximately $10-20 \%$ of individuals showed evidence of neurocysticercosis on computed tomography scans, primarily in the form of calcified lesions ${ }^{16-19}$. In some of these communities, the rate of epilepsy approaches 3\%, and approximately $30 \%$ of these cases showed evidence of cysticercosis. $^{17,20}$

Interestingly, in a Venezuelan community, a high percentage $(18.4 \%)$ of inpatients with chronic psychiatric disorders suffered from neurocysticercosis. ${ }^{21}$ However, individuals with cysticercosis, particularly with a history of migration, also search for medical assistance outside of endemic areas, where cysticercosis might be easily missed or only be identified accidentally. ${ }^{22-26}$ Furthermore, individuals without a history of eating pork or traveling to endemic areas have been described as experiencing neurocysticercosis. ${ }^{26}$ Epidemiologic studies have demonstrated that tight clustering in households and household contacts of patients with neurocysticercosis poses a threefold higher risk of positive serology for cysticercosis than controls. ${ }^{27}$

\section{Parasite Lifecycle}

The pork tapeworm $T$. solium belongs to the class of the Cestoda. The family Taeniidae is divided into three genera, of which $T$. solium is a species. ${ }^{28,29}$ The lifecycle of T. solium is complex and can contribute to several pathologies that affect both pigs and humans. The only definitive hosts within which the tapeworm can complete its lifecycle and survive in the form of adults are humans. ${ }^{11}$ Both humans and pigs have been reported as intermediate hosts in which tapeworm eggs can grow to the metacestode's larval stage. ${ }^{30}$ Taeniasis occurs after the ingestion of cysticerci-infected, undercooked pork. The larvae evaginate in the small intestine, and the head (scolex) attaches itself to the intestinal mucosa and starts developing segments (proglottides). T. solium has a scolex with four suckers with a double crown of hooks, a short body, and a wide strobila (measuring 2-4 m) consisting of several hundred proglottids. Gravid proglottides containing fertile eggs begin to detach from the distal end and are excreted in the feces around two months after infection. ${ }^{31}$ Such eggs are immediately infectious and do not need a developmental period outside the host. Humans get infected as intermediate hosts by ingesting food/water contaminated with feces that contain these eggs or proglottides or by person-toperson distribution. Humans are typically exposed to eggs via a fecal-oral transmission (eg, caused by poor hand hygiene); moreover, tapeworm carriers may also infect themselves. Upon the ingestion of eggs or proglottides, oncospheres hatch in the intestine, invade the intestinal wall, reach the bloodstream, and migrate over 60-70 days to various tissues and organs develop into cysticerci. Some cysticercosis will spread to the CNS, causing extreme neurocysticercosis (sequelae), Figure 2. The incidence of autoinfection is not known in individuals with taeniasis. However, up to $15 \%$ of neurocysticercosis patients, harbor a tapeworm, thus strongly suggesting autoinfection. $^{32,33}$

\section{Pathogenesis}

Cysticerci consist of two main parts: the vesicular wall and the scolex, the main body of the larva. ${ }^{34}$ After entering the brain, cysticerci are present in a vesicular form, the viable stage in which the parasites have a transparent membrane, a clear vesicular fluid, and a regular invaginated scolex. Cysticerci may remain viable for years. The colloidal stage, in which the vesicular fluid becomes turbid, is the first stage of cysticerci involution, and the scolex shows signs of hyaline degeneration. Subsequently, the cyst wall thickens and the scolex is converted into mineralized granules (the granular stage), after which the cysticercus is no longer viable. Finally, the parasite remnants appear as a mineralized nodule (calcified stage). ${ }^{35}$ In the underlying tissue, vesicular cysticerci elicit no inflammatory reaction.

By comparison, colloidal cysticerci are often surrounded by a capsule of collagen and a mononuclear inflammatory reaction involving the parasite itself, such as astrocytic gliosis, microglial proliferation, edema, degenerative neuronal changes, and perivascular lymphocyte infiltration of the surrounding brain parenchyma. This reaction could represent the basis for the neurological and psychiatric symptoms of neurocysticercosis. The edema subsides when parasites enter the granular and calcified stages, but the astrocytic changes can become more intense in the lesions' vicinity, ${ }^{36}$ Figure 3. Additionally, epithelioid cells emerge and coalesce to form multinucleated giant cells. $^{37}$ In a subarachnoid space, meningeal cysticerci naturally elicit a severe inflammatory reaction with the formation of an exudate comprising collagen fibers, lymphocytes, giant multinucleated cells, 


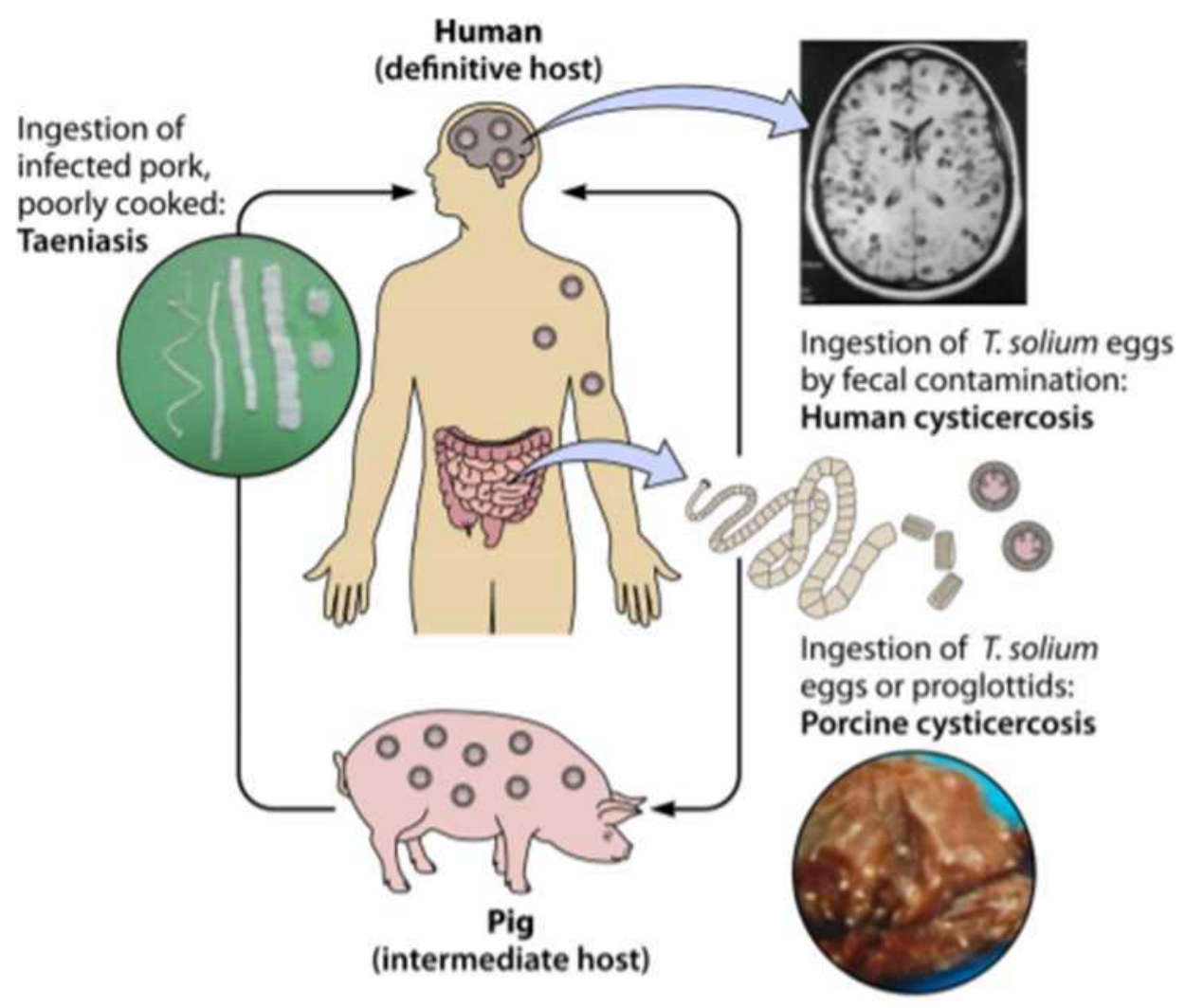

Figure 2 Life cycle of $T$. solium Development of both taeniasis and cysticercosis.

Notes: Reproduced from Garcia HH, Gonzalez AE, Gilman RH. Cysticercosis and Its Impact in Neurological Disease. Clin Microbiol Rev. 2020;33(3):e00085-I9. ${ }^{3}$ Copyright (C) 2020 American Society for Microbiology. Reproduced with permission from the American Society for Microbiology.
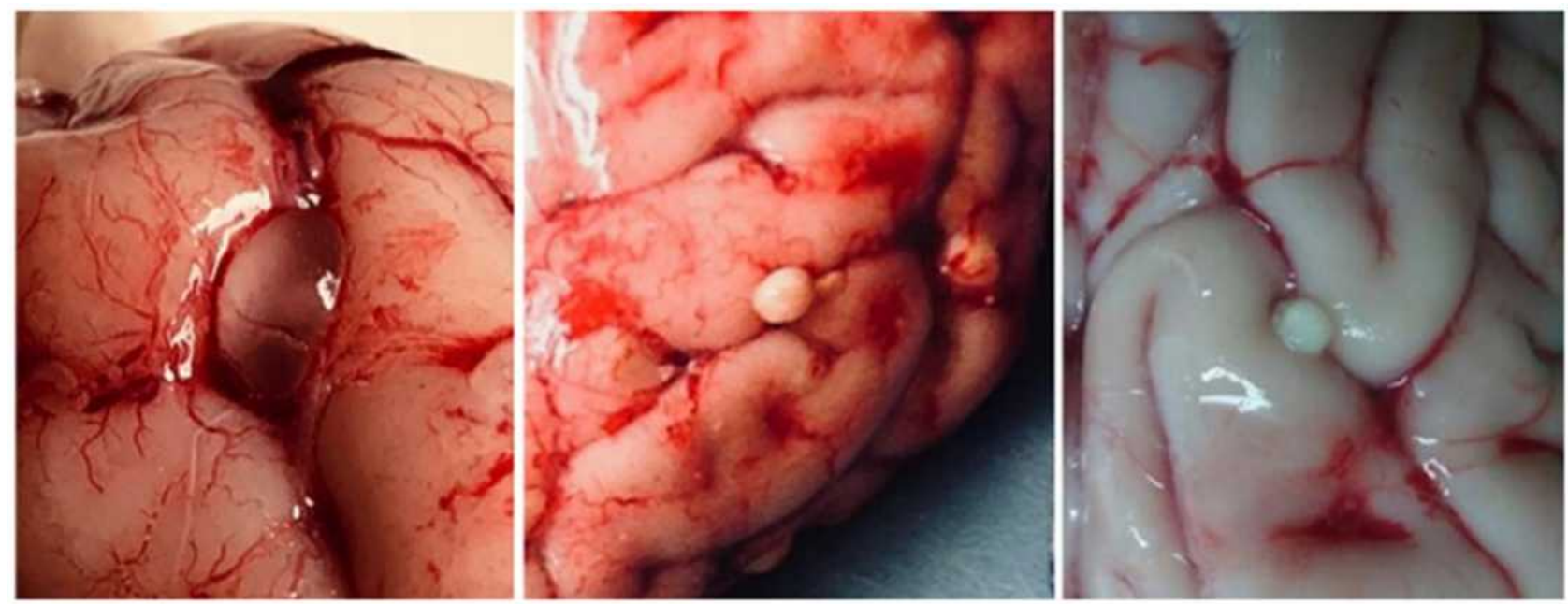

Figure 3 Macroscopic views of cysticerci in different stages of involution.

Notes: Reproduced from Garcia HH, O'Neal SE, Noh J, Handali S, Peru CWG in. Laboratory Diagnosis of Neurocysticercosis (Taenia solium). J Clin Microbiol. 20I8;56(9): e00424-18. ${ }^{36}$ Copyright (C) 2018 American Society for Microbiology. Reproduced with permission from the American Society for Microbiology.

eosinophils, and hyalinized parasitic membranes, further leading to the abnormal thickening of the leptomeninges. This inflammation will spread, further causing damage to the brain stem's optic chiasm and cranial nerves, as well as to small penetrating arteries emerging from the Willis' circle. The latter can induce the occlusion of the vessel's lumen with the subsequent development of cerebral infarction. ${ }^{38}$ Some cysticercal antigens stimulate the 
development of specific antibodies that form the basis of cysticercosis-related immunological diagnosis, whereas others (particularly antigen B) play a vital role in preventing the immune system from attacking cysticercosis. ${ }^{35}$ The T. solium cysticercus uses a series of active immune evasion mechanisms, which include protection by local barriers (such as the blood-brain barrier or the hemato-ocular barrier), blockage of the complement system, secretion of cytokines affecting the cellular response, safe degradation of host immunoglobulins, or masking itself with host immunoglobulins to evade immune surveillance. ${ }^{34,39}$

\section{Clinical Manifestation}

Depending on the cysts' location in the CNS, focal neurology or psychiatric symptoms manifest. Therefore, the following forms of neurocysticercosis have been defined:

\section{Parenchymatous Form}

Parenchymal neurocysticercosis is the most common type of neurocysticercosis. ${ }^{40}$ In the parenchymatic type, the parasite lodges as single or multiple cysts and forms clumps in the brain parenchyma. Pericystic inflammation results in the development of granuloma. The cysts can continue to develop and produce a space-occupying lesion. $^{41,42}$ This variety has an unfavorable prognosis as it may have a malignant path. The most common clinical manifestation is epilepsy.

\section{Meningeal Form}

The meningeal type is often associated with increased intracranial pressure in approximately $16 \%$ of the cases $^{9,43,44}$ secondary to widespread subarachnoiditis, arachnoiditis, and cerebrospinal fluid (CSF) obstruction resulting in hydrocephalus with clinical presentation of headache, intracranial hypertension, and disturbance of consciousness. ${ }^{4-47}$ Due to vasculitis and fibrous cranial nerves entrapment, it can also manifest as focal neurological deficits. $^{48}$

\section{Intraventricular and Subarachnoid Forms}

The intraventricular and subarachnoid forms of neurocysticercosis are observed in 15-54\% of patients and are more aggressive in clinical terms than the parenchymatic form. ${ }^{49-51}$ Here, the parasite migrates through the ventricular system, occluding contact corridors for CSF, causing acute ventriculomegaly episodes leading to sudden death or focal compression mass effects. Approximately $38 \%$ of patients demonstrate a rapid clinical decline. Larval death causes epididymitis and ventricular outlet occlusion, thus leading to hydrocephalus. ${ }^{51}$ Patients often have an elevated intracranial pressure due to a large cyst size or load, CSF pathway occlusion, associated epididymitis, and basal arachnoiditis. ${ }^{50}$

\section{Spinal Form}

The spinal form occurs in 1.6-13\% of patients with neurocysticercosis. There are two known types of spinal neurocysticercosis: extra-medullary and intra-medullary. This form typically occurs due to the downward migration of larvae from the cerebral to the spinal subarachnoid space. $^{30,52}$

\section{Neurological Symptoms}

The concept of a standard neurocysticercosis clinical syndrome is not feasible. The disorder may be asymptomatic or may manifest with a range of non-specific symptoms, such as seizures, headaches, elevated intracranial pressure, or cognitive impairment. ${ }^{44,53}$ This pleomorphism is predominantly associated with individual variations in the number and location of nervous system lesions and the magnitude of the host's immune response to the parasite. ${ }^{54}$ One of the remarkable aspects of neurocysticercosis is that a high proportion of individuals with parasites in the CNS remain free of symptoms. In other individual patients, either inflammation around the parasite or mass effects produce clinical manifestations only several years after the parasite lodges in the CNS. ${ }^{12}$ As mentioned above, neurological symptoms and particularly epileptic seizures, are regarded as the most common neurocysticercosis manifestation. In $50-80 \%$ of patients with parenchymal brain cysts or calcifications, seizures occur but are less frequent in other forms. Headaches, hydrocephalus, chronic meningitis, focal neurological deficits are other frequently observed characteristics. ${ }^{55-57}$ Neurocysticercosis also presents with intracranial hypertension, hydrocephalus, or both in $20-30 \%$ of cases. ${ }^{50,58}$ Further, brainstem dysfunction manifestations, cerebellar ataxia, sensory deficits, involuntary movements, stroke-like symptoms, extrapyramidal signs, and dementia have been described among patients with neurocysticercosis. ${ }^{55}$

\section{Psychiatric Manifestations}

Very few publications, mostly anecdotal accounts from South America or brief explanations of clinical cases in neurological research, have considered neurocysticercosis's psychiatric symptoms. ${ }^{19,21,52,59-72}$ Psychological 
effects of neurocysticercosis have been particularly detailed in the form of a case series from the early 20th century, documenting therapeutic and pathological elements. ${ }^{73}$ Besides, various studies were conducted in the first half of the century in psychiatric hospitals, in which detailed clinical and pathological definitions can be found ${ }^{74}$ this time medical surveys focusing on patients attending the Neurologic Clinic of the University of São Paulo Medical School reported up to $75 \%$ of severe mental disorders associated with neurocysticercosis. ${ }^{74}$ More than $15 \%$ of patients with neurocysticercosis develop psychotic disorders. $^{71}$ Of historical significance, the first case recorded from Asia of neurocysticercosis came from the autopsy of an inmate patient in a lunatic asylum in Madras. ${ }^{9}$ A variety of mental problems induced by neurocysticercosis, such as delirium, perceptive sensory changes, anxiety, depression, and personality disorders, have been identified. Recent studies have shown that many patients with neurocysticercosis show a decline in the quality of life, depression, or moderate cognitive impairment that is not explicitly connected to the disease's severity or a particular type. ${ }^{34,59,70}$ The presence of elevated intracranial pressure is associated with these symptoms but not comorbidity with parenchymal brain cysts or seizures. ${ }^{75}$ Particularly in patients with parenchymal neurocysticercosis, psychotic episodes characterized by confusion, delusional ideation, psychomotor agitation, aggressive behavior, and visual hallucinations have been identified. ${ }^{62,66}$ Here, we will discuss the most common psychiatric manifestations that have been observed in neurocysticercosis, Box 1.

\section{Cognitive Functions}

In 1995, Levav et al conducted the first study using structured cognitive testing in patients with neurocysticercosis and controls. The authors recruited a representative sample of patients from a broader epidemiological investigation on epilepsy and neurocysticercosis in Ecuador. In total,

Box I An Overview of Psychiatric Manifestations in Neurocysticercosis

\begin{tabular}{|l|}
\hline Psychiatric Disorders in Association with Neurocysticercosis \\
\hline I-Cognitive dysfunction \\
2- Depression/Mixed anxiety depression \\
3- Dementia \\
4-Psychosis \\
5-Personality changes \\
\hline
\end{tabular}

123 subjects agreed to participate in their study. ${ }^{76}$ The research found that patients with neurocysticercosis had significant deficits regarding attention and motor functioning relative to controls.

In a case series from Brazil, Forlenza et al reported neurocysticercosis's psychiatric and cognitive manifestations. In their cross-sectional study, the extent and characteristics of psychiatric morbidity were assessed in 38 outpatients with reported neurocysticercosis. Psychiatric diagnoses were made using the present state examination and the schedule for affective disorders and schizophrenia - lifetime version; the cognitive status was evaluated by a mini-mental state examination and Strub and Black's mental status examination. A mild to moderate cognitive impairment was observed in $84 \%$ of the cases using Strub and Black's mental status examination, while only $26 \%$ of the cases achieved the maximum score of 30 in the Minimental status examination. ${ }^{75}$ Over one year, a total of 90 patients with untreated neurocysticercosis from the clinical divisions of the National Institute of Neurology and Neurosurgery of Mexico City were included in a study by Ramirez-Bermudez et al They announced that 20 patients $(22.2 \%)$ had mild forms of cognitive impairment as shown by direct mental assessment (COGNISTAT). ${ }^{60}$ Recently, Ciampi De Andrade et al presented a report on cognitive changes and neurocysticercosis. ${ }^{59}$ The authors report the inclusion of 45 non-immigrant patients younger than 60 years with active neurocysticercosis (ie, at least one MRI scolex cystic lesion) in the sample, thereby meeting the absolute requirement for confirmed neurocysticercosis by finding the Larvae in the CNS. In total, 28 patients with cryptogenic epilepsy from their institution were subjected to cognitive testing. The patients with $\mathrm{CE}$ were diagnosed according to the guidelines of the International League Against Epilepsy. Compared to stable controls and epilepsy controls, neurocysticercosis cases displayed substantial impairment in executive performance, memory, positive practice, and verbal fluency.

Rodrigues et al assessed 40 treatment-naive patients with calcified neurocysticercosis (C-NC) aged $37.6 \pm$ 11.3 years. The adherence to conclusive $\mathrm{C}-\mathrm{NC}$ requirements was subjected to a rigorous cognitive and functional assessment, and 40 active patients with $\mathrm{NC}$ and 40 healthy controls (HC) were matched for age and education. Cognitive dysfunction was present in 10 patients $(25 \%) .{ }^{61,77}$ To observe cognitive and behavioral changes, Prasad et al conducted their study on three different children's groups. The first group was 83 reported cases of 
confirmed neurocysticercosis, which were diagnosed according to the updated Del Brutto criteria (Group-1). ${ }^{19}$ Such criteria are briefly divided into absolute and clinical/ exposure criteria. Absolute criteria include parasite histological confirmation, proof of subretinal cysts, and scolex demonstration within a cyst. Neuroimaging conditions are classified as major, confirmatory and minor; Major signs in neuroimaging include cystic lesions without scolex, lesion enhancement, multilobulated cysts, and calcifications). Confirmatory signs include (including cyst resolution after cystic drug therapy, spontaneous resolution of single enhancing lesions, and migratory ventricular cysts in sequential neuroimaging studies), while minor (hydrocephalus and leptomeningeal enhancement).

Clinical/exposure criteria include the serological evidence of infection by detecting anticysticercal antibodies or cysticercal antigens, systemic cysticercosis, confirmation of a Taenia solium household carrier, suggestive clinical symptoms, and residence in endemic regions. There were two other control groups: children with generalized idiopathic tonic-clonic seizures (Group II) and otherwise healthy children with non-specific cough (Group III). A cognitive and behavioral examination of cases and controls revealed substantial differences in visual perception, immediate memory recall, analysis, verbal capacity, memory, and spatial ability. In older children, neurocysticercosis leads to a drop in cognitive function. ${ }^{18}$ Varghese et al 2016 conducted a prospective, hospital-based study at a Tertiary Referral Centre in India from April 2012 to December 2013. According to diagnostic criteria, 43 patients chosen for the study were diagnosed with definite or probable neurocysticercosis. The researchers found memory problems in $27.9 \%$ of cases. ${ }^{64}$ Medina-Ortega et al documented a case of a 51-year-old male patient from the rural region of Caldono (Cauca, Colombia). The patient had recurrent cognitive impairment and associated neuropsychiatric manifestations triggered by recurrent neurocysticercosis. ${ }^{69}$ Some reports have shown favorable outcomes in patients with cognitive impairment and neurocysticercosis after appropriate therapy. ${ }^{52,79}$ On a neuropathological level, multiple factors functioning alone or in conjunction including local inflammatory (parasitic) reactions, vascular lesions, immune-mediated reactions, or secondary epilepsy induced by neurocysticercosis, as effects of anti-epileptic drugs, may trigger cognitive impairment. ${ }^{67}$ It has been suggested that in patients with vulnerable brains, frontal-parietal-temporal network functioning might be impaired, as it correlated with cognitive functioning, which was measured using Minimental State Examination and Neurobehavioral Cognitive Status Examination. ${ }^{60}$ Executive functions have been particularly reported to be affected by the infestation of cysticercosis. Executive functions are cognitive mechanisms that underlie the ability to respond to complex circumstances and their organizational controls adaptively. ${ }^{80}$

\section{Depression}

Psychiatric abnormalities, especially depressive syndromes, are common in patients with neurocysticercosis. As reported by Flourenza et al, the most common psychiatric diagnosis $(52.6 \%)$ among 38 outpatients with neurocysticercosis was depression. Psychiatric diagnoses were made using the present state examination and the schedule for affective disorders and schizophrenia-lifetime version. Higher clinical morbidity was associated with active disease and intracranial hypertension, and a history of mood disorders was strongly associated with present depression. ${ }^{75,81}$ A different Brazil study observed a correlation between neurocysticercosis and depression in 114 outpatients, subdivided into four groups: NCC with epilepsy, NCC without epilepsy, epilepsy without NCC and chronic headache. The authors report that the prevalence of depression in the examined patients was higher than the general population. The proportion of patients with depression was as follows: epilepsy + neurocysticercosis $=83 \%$ and epilepsy-free neurocysticercosis $=88 \%$.

Moreover, psychotic disorders were less common than previously recorded; however, this may again be clarified by using a study of neurological outpatients that are likely to have less severe psychiatric disorders. ${ }^{68}$ Ciampi de Andrade et al (previously mentioned in the psychiatric manifestations section) examined 40 treatment-naive patients with NCYST and found that depression was evident in $27.5 \%$ of patients with active neurocysticercosis. ${ }^{59}$ Srivastava et al took detailed psychiatric history and conducted a detailed clinical examination of 50 patients with epilepsy and neurocysticercosis and 50 patients with epilepsy and no proof of neurocysticercosis. ${ }^{82}$ They used a semistructured interview to assess psychiatric symptoms using the Comprehensive Psychopathology Rating Scale. They found clinical morbidity in $68 \%$ of patients with epilepsy and neurocysticercosis, which was substantially higher than that in the control group of patients with epilepsy and without neurocysticercosis. In both cases (36\%) and controls (30\%), severe depression was the 
most common co-diagnosis, followed by mixed anxiety depression diagnosed in $18 \%$ and $10 \%$ of the cases and controls, respectively. The pathophysiology of how neurocysticercosis infection leads to major depressive episodes remains unknown. ${ }^{83}$ However, it seems essential to assess the link between immunologic response and incidence of depression in neurocysticercosis ${ }^{83}$ as there is a clear indication that cytokines play an important role in depression. $^{83}$

\section{Dementia}

Dementia has been described as a neuropsychiatric presentation of neurocysticercosis with a unique clinical feature - it is reversible and patients may fully recover, as reported by Machado-Porto et al. in a 63 -year-old woman with racemose neurocysticercosis. ${ }^{84,85}$ Other than degenerative dementia with its higher prevalence in the elderly, neurocysticercosis-related dementia is observed in any age group. Neurocysticercosis-related dementia may represent the only manifestation of the infection, but dementia may also co-manifest with other neuropsychiatric symptoms like hallucinations and neurological presentations like seizures. ${ }^{84,86}$ Around one-fifth of patients with neurocysticercosis are confirmed to have dementia. ${ }^{87}$ Nonetheless, the exact prevalence of neurocysticercosis is seldom reported in patients with psychiatric disorders, which may be due to the lack of concern about neurocysticercosis as a causative agent of psychological manifestation.
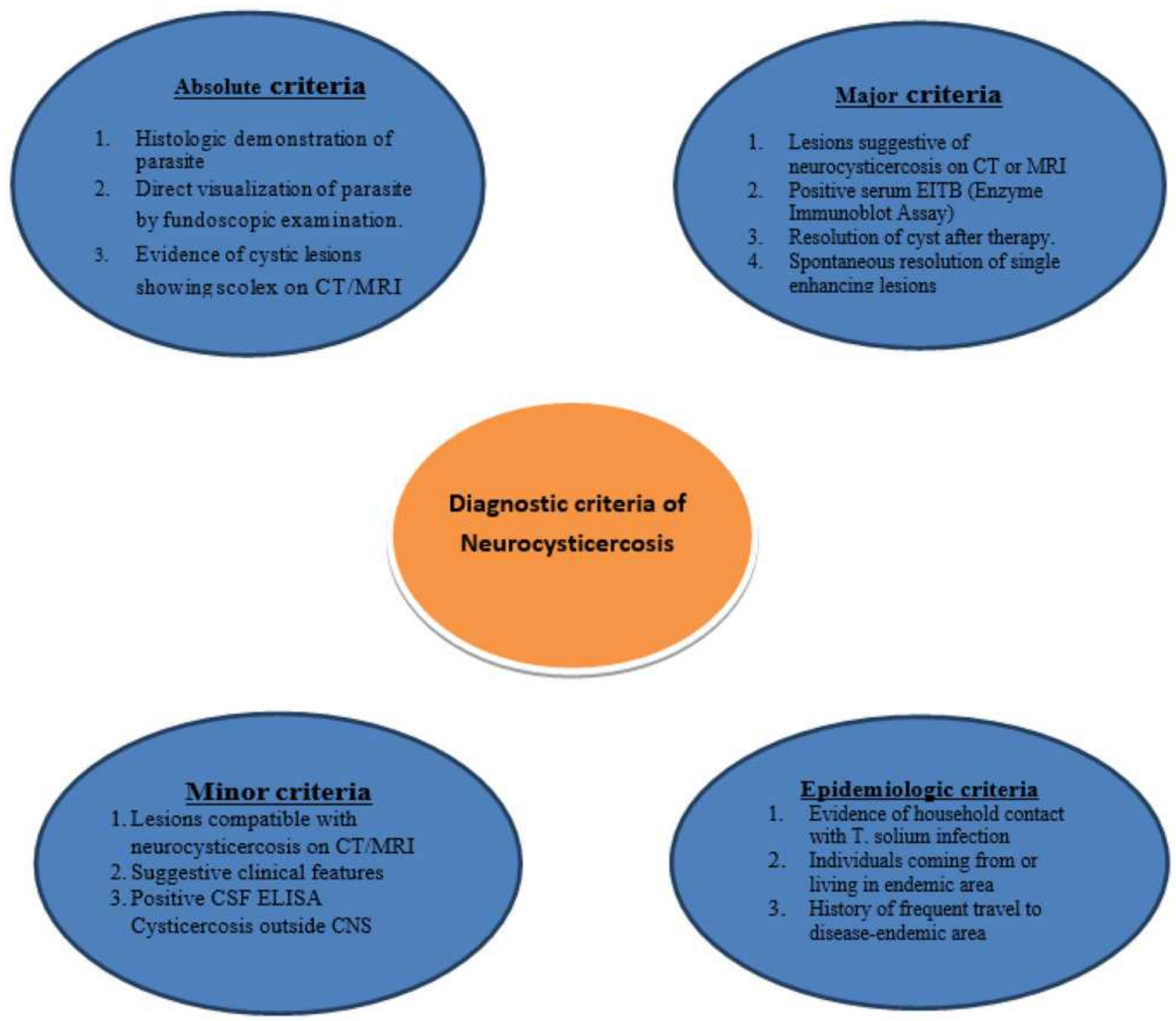

Figure $4 \mathrm{~A}$ drawing showing the diagnostic criteria for neurocysticercosis.

Note: These criteria were established by Del Brutto et al; data from Del Brutto et al. ${ }^{78}$ 
Approximately one-fifth of hospitalized patients with psychiatric disorders in the state of Tachira (Venezuela) (patients in the psychiatric department) reported seropositivity to cysticercosis, thus emphasizing dementia. ${ }^{21}$ Ramirez-Bermudez et al researched dementia in neurocysticercosis. The authors determined that 82 patients with a definitive or likely neurocysticercosis diagnosis had active and inactive manifestations of the disease. Dementia was found in $17 \%$ of patients. In this analysis, some facts may account for a slightly greater prevalence of dementia. ${ }^{60}$ Patients with mental disorders may also be at an elevated risk of developing neurocysticercosis because they may become infected by contact with feces due to bad hygiene practices. Neurocysticercosis can be responsible for most major psychiatric syndromes (such as schizophrenic and affective disorders) and dementia. ${ }^{63}$

According to the literature, psychosis is a rare presentation in Neurocysticercosis and might present in up to $5 \%$ of patients ${ }^{88}$. The reported psychosis cases in the literature were mainly case reports. Mixed anxiety depression was also reported in a previous study of neurocysticercosis patients. $^{2}$ Antisocial personality changes have been also reported previously in patients with neurocysticercosis. We now added those psychiatric disorders to the revised manuscript. ${ }^{89}$

\section{Diagnosis}

Neurocysticercosis diagnosis is based on various observations, including, if available, epidemiology, symptoms, signs, serology, and pathology (Figure 4). Definite diagnoses require brain imaging.

\section{Imaging}

Neuroimaging is essential to diagnose cysticercosis to assess the form of the disease and required emergency measures. To identify affected brain regions as well as active cysts, MRI is superior to CT scans. However, CT is superior for the detection of calcifications. CT imaging is ideal for detecting calcification and a complete MRI analysis is necessary to identify subarachnoid and ventricular manifestations of the disease. ${ }^{90,91}$

\section{Serology}

Several serological tests that measure antibodies against T. Solium have been developed over time, Box 2. A serum Western blot employing a particular fraction of T. solium is the best known and most useful test. This test particularly confirms the diagnosis of taeniasis/cysticercosis. ${ }^{34,92-95}$
Box 2 Summarization of the Commonly Used Immunological Tests for Diagnosis of Neurocysticercosis. ${ }^{97,98}$

\begin{tabular}{|c|c|}
\hline ImmunoBlot & ELISA \\
\hline $\begin{array}{l}\text { - The current choice for serolo- } \\
\text { gical diagnosis of } \\
\text { neurocysticercosis } \\
\text { - CDC's immunoblot is based on } \\
\text { detection of antibody to one } \\
\text { or more of } 7 \text { lentil-lectin puri- } \\
\text { fied structural glycoprotein } \\
\text { antigens from the larval cysts of } \\
\text { T. solium. } \\
\text { - In clinical settings, the diagnos- } \\
\text { tic performance of the LLGP- } \\
\text { EITB performed in serum sam- } \\
\text { ples is very high, approaching } \\
98 \% \text { sensitivity and I00\% spe- } \\
\text { cificity in patients with more } \\
\text { than one viable brain cysticer- } \\
\text { cosis cyst. } \\
\text { - In patients with a single viable } \\
\text { or degenerating cyst, the sen- } \\
\text { sitivity is lower (60\% to } 70 \% \text { ). }\end{array}$ & $\begin{array}{l}\text { - Low specificity of the test has } \\
\text { been recognized as a major } \\
\text { problem because of cross- } \\
\text { reaction of the components of } \\
\text { the crude antigens of } \\
\text { cysticerci. } \\
\text { - These components cross react } \\
\text { with antibodies specific for } \\
\text { many helminthic infections, } \\
\text { especially echinococcosis and } \\
\text { filariasis. }\end{array}$ \\
\hline
\end{tabular}

The major disadvantage is that it lacks sensitivity in the patients with low disease burden as those with one viable or degenerating cyst. Moreover, it may be negative in the patients with only calcified lesions. ${ }^{96}$

Antigen detection tests have been developed using monoclonal antibodies in an ELISA capture format. They are specific for the diagnosis of possible infection but are not as sensitive as antibody assays. In patients with the subarachnoid disease, the antigen is usually present but is less common in the parenchymal disease. The antigen concentration is greater than the serum in the CSF. Levels correlate grossly with the degree of involvement. ${ }^{61}$

\section{Conclusion}

Although neurocysticercosis is the most widespread parasitic CNS disease worldwide, ${ }^{59,99}$ its psychiatric manifestations do not appear to be well researched in the literature. Therefore, this narrative review aimed to highlight the various psychiatric symptoms of neurocysticercosis.

Although data are available about this disorder from the early 20th century, much room is available for future research. Retrospective, prospective cohort studies, more population-based neuroimaging studies, and crosssectional studies could help regarding a more accurate estimation of the prevalence of neurocysticercosis. 
Immigrants from endemic areas presenting with psychiatric symptoms or treatment-resistant depression should receive brain imaging to exclude neurocysticercosis as a potential organic etiology. The correlation between the presenting psychopathology and the localization of the brain's cysts could help neuroscientists better understand the affected brain corresponding to specific psychiatric symptoms.

\section{Disclosure}

The authors reported no conflicts of interest for this work.

\section{References}

1. Del Brutto OH. Neurocysticercosis: a review. ScientificWorldJournal. 2012;2012:159821. doi:10.1100/2012/159821

2. Tavares AR. Psychiatric disorders in neurocysticercosis. $\mathrm{Br}$ J Psychiatry. 1993;163(DEC.):839. doi:10.1192/bjp.163.6.839a

3. Del Brutto OH, Sotelo J. Neurocysticercosis: an update. Rev Infect Dis. 1988;10(6):1075-1087. doi:10.1093/clinids/10.6.1075

4. Cox FEG. History of human parasitology. Clin Microbiol Rev. 2002;15(4):595LP- 612. doi:10.1128/CMR.15.4.595-612.2002

5. Ignacio Olivé J, Angulo-Rivero P. Cysticercosis of the nervous system I. Introduction and general aspects. J Neurosurg. 1962;19 (8):632-634. doi:10.3171/jns.1962.19.8.0632

6. Flisser A. Neurocysticercosis in Mexico. Parasitol Today. 1988;4 (5):131-137. doi:10.1016/0169-4758(88)90187-1

7. St S. Uber einen Cysticercus cellulosae im Men- schlichen Auge. Oken's Iseis. 1830;8:717-718.

8. Foyaca Sibat H, Ibañez Valdeès LF. Introduction to Cysticercosis and Its Historical Background. In: Novel Aspects on Cysticercosis and Neurocysticercosis. 2013;3-38. doi:10.5772/50318

9. Del Brutto OH, García HH. Cysticercosis of the Human Nervous System. 1st ed. Springer-Verlag Berlin Heidelberg; 2014. doi:10.1007/978-3-642-39022-7

10. Winkler AS. Neurocysticercosis in sub-Saharan Africa: a review of prevalence, clinical characteristics, diagnosis, and management. Pathog Glob Health. 2012;106(5):261-274. doi:10.1179/2047 773212Y.0000000047

11. García HH, Del Brutto OH, Casulli A. Fake news in neglected tropical diseases: the case of neurocysticercosis. PLoS Negl Trop Dis. 2020;14(6):1-2. doi:10.1371/journal.pntd.0008208

12. White ACJ. Neurocysticercosis: updates on epidemiology, pathogenesis, diagnosis, and management. Annu Rev Med. 2000;51 (1):187-206. doi:10.1146/annurev.med.51.1.187

13. Garcia HH, Gonzalez AE, Gilman RH. Taenia solium cysticercosis and its impact in neurological disease. Clin Microbiol Rev. 2020;33 (3):e00085-19. doi:10.1128/CMR.00085-19

14. Debacq G, Moyano LM, Garcia HH, et al. Systematic review and meta-analysis estimating association of cysticercosis and neurocysticercosis with epilepsy. PLoS Negl Trop Dis. 2017;11(3):e0005153. doi:10.1371/journal.pntd.0005153

15. Del Brutto OH, Arroyo G, Del Brutto VJ, Zambrano M, García HH. On the relationship between calcified neurocysticercosis and epilepsy in an endemic village: a large-scale, computed tomography-based population study in rural Ecuador. Epilepsia. 2017;58 (11):1955-1961. doi:10.1111/epi.13892

16. Fleury A, Gomez T, Alvarez I, et al. High prevalence of calcified silent neurocysticercosis in a rural village of Mexico. Neuroepidemiology. 2003;22(2):139-145. doi:10.1159/000068 748
17. Moyano LM, Saito M, Montano SM, et al. Neurocysticercosis as a cause of epilepsy and seizures in two community-based studies in a cysticercosis-endemic region in Peru. PLoS Negl Trop Dis. 2014;8 (2):e2692. doi:10.1371/journal.pntd.0002692

18. Prasad R, Shambhavi MOP, Upadhyay SK, Singh TB, Singh UK. Cognitive and behaviour dysfunction of children with neurocysticercosis: a Cross-Sectional Study. J Trop Pediatr. 2014;60(5):358-362. doi:10.1093/tropej/fmu029

19. Prasad KN, Verma A, Srivastava S, Gupta RK, Pandey CM, Paliwal VK. An epidemiological study of asymptomatic neurocysticercosis in a pig farming community in northern India. Trans $R$ Soc Trop Med Hyg. 2011;105(9):531-536. doi:10.1016/j.trstmh. 2011.06.001

20. Rajshekhar V, Raghava MV, Prabhakaran V, Oommen A, Muliyil J. Active epilepsy as an index of burden of neurocysticercosis in Vellore district, India. Neurology. 2006;67(12):2135-2139. doi:10.1212/01. wnl.0000249113.11824.64

21. Meza NW, Rossi NE, Galeazzi TN, et al. Cysticercosis in chronic psychiatric inpatients from a Venezuelan community. Am J Trop Med Hyg. 2005;73(3):504-509. doi:10.4269/ajtmh.2005.73.504

22. Croker C, Redelings M, Reporter R, Sorvillo F, Mascola L, Wilkins P. The impact of neurocysticercosis in california: a review of hospitalized cases. PLoS Negl Trop Dis. 2012;6(1):e1480. doi:10.1371/journal.pntd.0001480

23. O'Neal SE, Flecker RH. Hospitalization frequency and charges for neurocysticercosis, United States, 2003-2012. Emerg Infect Dis. 2015;21(6):969-976. doi:10.3201/eid2106.141324

24. Ong S, Talan DA, Moran GJ, et al. Neurocysticercosis in radiographically imaged seizure patients in U.S. emergency departments. Emerg Infect Dis. 2002;8(6):608-613. doi:10.3201/eid0806.010377

25. Schantz PM, Moore AC, Muñoz JL, et al. Neurocysticercosis in an Orthodox Jewish community in New York City. $N$ Engl $J$ Med. 1992;327(10):692-695. doi:10.1056/NEJM199209033271004

26. Sorvillo FJ, DeGiorgio C, Waterman SH. Deaths from cysticercosis, United States. Emerg Infect Dis. 2007;13(2):230-235. doi:10.3201/ eid1302.060527

27. Lescano AG, Garcia HH, Gilman RH, et al. Taenia solium cysticercosis hotspots surrounding tapeworm carriers: clustering on human seroprevalence but not on seizures. PLoS Negl Trop Dis. 2009;3(1): e371. doi:10.1371/journal.pntd.0000371

28. Nakao M, Lavikainen A, Yanagida T, Ito A. Phylogenetic systematics of the genus Echinococcus (Cestoda: Taeniidae). Int $J$ Parasitol. 2013;43(12-13):1017-1029. doi:10.1016/j. ijpara.2013.06.002

29. Bobes RJ, Fragoso G, Fleury A, et al. Evolution, molecular epidemiology and perspectives on the research of taeniid parasites with special emphasis on Taenia solium. Infect Genet Evol. 2014;23:150-160. doi:10.1016/j.meegid.2014.02.005

30. Coral-Almeida M, Gabriël S, Abatih EN, Praet N, Benitez W, Dorny P. Taenia solium human cysticercosis: a systematic review of sero-epidemiological data from endemic zones around the world. PLoS Negl Trop Dis. 2015;9(7):e0003919. doi:10.1371/journal. pntd.0003919

31. Flisser A. Taeniasis and cysticercosis due to Taenia solium. Prog Clin Parasitol. 1994;4:77-116.

32. García HH, Del Brutto OH. Heavy nonencephalitic cerebral cysticercosis in tapeworm carriers. The Cysticercosis Working Group in Perú. Neurology. 1999;53(7):1582-1584. doi:10.1212/wnl.53.7.1582

33. Greene RM, Hancock K, Wilkins PP, Tsang VC. Taenia solium: molecular cloning and serologic evaluation of $14-$ and $18-\mathrm{kDa}$ related, diagnostic antigens. J Parasitol. 2000;86(5):1001-1007. doi:10.1645/0022-3395(2000)086[1001:TSMCAS]2.0.CO;2

34. Willms K. Morphology and biochemistry of the pork tapeworm, Taenia solium. Curr Top Med Chem. 2008;8(5):375-382. doi:10. $2174 / 156802608783790875$ 
35. Flisser A, Correa DEC. "The Pathology Of Neurocysticercosis," in Taenia Solium Cysticercosis. From Basic to Clinical Science. (S Prabhakar and G. Singh, ed.). 1st ed. Oxford University Press; December 19, 2002.

36. Garcia HH, O'Neal SE, Noh J, Handali S. Peru CWG in. Laboratory diagnosis of neurocysticercosis (Taenia solium). J Clin Microbiol. 2018;56(9):e00424-18. doi:10.1128/JCM.00424-18

37. Pittella JEH. Neurocysticercosis. Brain Pathol. 1997;7(1):681-693. doi:10.1111/j.1750-3639.1997.tb01083.x

38. Del Brutto OH. Stroke and vasculitis in patients with cysticercosis. In: Bogousslavsky J, Caplan LR, editors. Uncommon Causes of Stroke. 2nd ed. Cambridge University Press;2008: 53-58. doi:10.1017/CBO9780511544897.010

39. Prodjinotho UF, Lema J, Lacorcia M, et al. Host immune responses during Taenia solium neurocysticercosis infection and treatment. PLoS Negl Trop Dis. 2020;14(4):e0008005. doi:10. 1371/journal.pntd.0008005

40. Venkat B, Aggarwal N, Makhaik S, Sood R. A comprehensive review of imaging findings in human cysticercosis. Jpn J Radiol. 2016;34 (4):241-257. doi:10.1007/s11604-016-0528-4

41. Aggarwal A, Aneja S, Taluja V, Kumar R, Bhardwaj K. Etiology of partial epilepsy. Indian Pediatr. 1998;35:49-52.

42. Singhal BS, Ladiwala U, Singhal P. Neurocysticercosis in the Indian context: with special reference to solitary parenchymatous cyst. Neurol India. 1997;45(4):211-217.

43. Garcia HH, Nash TE, Del Brutto OH. Clinical symptoms, diagnosis, and treatment of neurocysticercosis. Lancet Neurol. 2014;13 (12):1202-1215. doi:10.1016/S1474-4422(14)70094-8

44. Carabin H, Ndimubanzi PC, Budke CM, et al. Clinical manifestations associated with neurocysticercosis: a systematic review. PLoS Negl Trop Dis. 2011;5(5):e1152. doi:10.1371/journal.pntd.0001152

45. Torres-Corzo JG, Tapia-Pérez JH, Vecchia RR-D, Chalita-Williams JC, Sánchez-Aguilar M, Sánchez-Rodríguez JJ. Endoscopic management of hydrocephalus due to neurocysticercosis. Clin Neurol Neurosurg. 2010;112(1):11-16. doi:10.1016/j.clineuro.2009.08.022

46. Santo AH. Cysticercosis-related mortality in the State of São Paulo, Brazil, 1985-2004: a study using multiple causes of death. Cad Saude Publica. 2007;23(12):2917-2927. doi:10.15 90/s0102-311×2007001200013

47. Fleury A, Carrillo-Mezo R, Flisser A, Sciutto E, Corona T. Subarachnoid basal neurocysticercosis: a focus on the most severe form of the disease. Expert Rev Anti Infect Ther. 2011;9(1):123-133. doi:10.1586/eri.10.150

48. Joubert J. Cysticercal meningitis-a pernicious form of neurocysticercosis which responds poorly to praziquantel. S Afr Med J. 1990;77 (10):528-530.

49. Zee CS, Segall HD, Destian S, Ahmadi J, Apuzzo ML. MRI of intraventricular cysticercosis: surgical implications. J Comput Assist Tomogr. 1993;17(6):932-939. doi:10.1097/00004728-1993 11000-00015

50. Lobato RD, Lamas E, Portillo JM, et al. Hydrocephalus in cerebral cysticercosis. Pathogenic and therapeutic considerations. $J \quad$ Neurosurg. 1981;55(5):786-793. doi:10.3171/jns.1981.55.5.0 786

51. Apuzzo ML, Dobkin WR, Zee CS, Chan JC, Giannotta SL, Weiss MH. Surgical considerations in treatment of intraventricular cysticercosis. An analysis of 45 cases. J Neurosurg. 1984;60 (2):400-407. doi:10.3171/jns.1984.60.2.0400

52. Sharma S, Modi M, Lal V, Prabhakar S, Bhardwaj A, Sehgal R. Reversible dementia as a presenting manifestation of racemose neurocysticercosis. Ann Indian Acad Neurol. 2013;16(1):88-90. doi:10.4103/0972-2327.107706

53. Del Brutto OH. A review of cases of human cysticercosis in Canada. Can J Neurol Sci. 2012;39(3):319-322. doi:10.1017/ s0317167100013445
54. Fleury A, Escobar A, Fragoso G, Sciutto E, Larralde C. Clinical heterogeneity of human neurocysticercosis results from complex interactions among parasite, host and environmental factors. Trans $R$ Soc Trop Med Hyg. 2010;104(4):243-250. doi:10.1016/j. trstmh.2010.01.005

55. Carpio A. Neurocysticercosis: an update. Lancet Infect Dis. 2002;2 (12):751-762. doi:10.1016/s1473-3099(02)00454-1

56. Chandramuki A, Nayak P. Sub acute and chronic meningitis in children - an immunological study of cerebrospinal fluid. Indian J Pediatr. 1990;57(5):685-691. doi:10.1007/BF02728714

57. David S, Mathai E. Ocular cysticercosis-a review of 25 cases. J Assoc Physicians India. 2000;48(7):704-707.

58. Sotelo J, Guerrero V, Rubio F. Neurocysticercosis: a new classification based on active and inactive forms. A study of 753 cases. Arch Intern Med. 1985;145(3):442-445. doi:10.1001/archinte. 1985.00360030074016

59. Ciampi De Andrade D, Rodrigues CL, Abraham R, et al. Cognitive impairment and dementia in neurocysticercosis: a cross-sectional controlled study. Neurology. 2010;74(16):1288-1295. doi:10.1212/ WNL.0b013e3181d9eda6

60. Ramirez-Bermudez J, Higuera J, Sosa AL, Lopez-Meza E, LopezGomez M, Corona T. Is dementia reversible in patients with neurocysticercosis? J Neurol Neurosurg Psychiatry. 2005;76 (8):1164-1166. doi:10.1136/jnnp.2004.052126

61. Rodriguez S, Dorny P, Tsang VCW, et al. Detection of Taenia solium antigens and anti-T. solium antibodies in paired serum and cerebrospinal fluid samples from patients with intraparenchymal or extraparenchymal neurocysticercosis. J Infect Dis. 2009;199(9):1345-1352. doi:10.1086/597757

62. Shandera WX, White ACJ, Chen JC, Diaz P, Armstrong R. Neurocysticercosis in Houston, Texas. A report of 112 cases. Medicine. 1994;73(1):37-52. doi:10.1097/00005792-19940100000004

63. Scharf D. Neurocysticercosis: two hundred thirty-eight cases from a California Hospital. Arch Neurol. 1988;45(7):777-780. doi:10.1001/archneur.1988.00520310087022

64. Varghese V, Chandra SR, Christopher R, et al. Cognitive dysfunction and its determinants in patients with neurocysticercosis. Indian J Psychol Med. 2016;38(2):142-146. doi:10.4103/0253-7176.178809

65. Wallin MT, Pretell EJ, Bustos JA, et al. Cognitive changes and quality of life in neurocysticercosis: a longitudinal study. PLoS Negl Trop Dis. 2012;6(1):e1493. doi:10.1371/journal.pntd.0001493

66. Shriqui CL, Milette PC. You drive me crazy: a case report of acute psychosis and neurocysticercosis. Can J Psychiatry. 1992;37 (2):121-124. doi:10.1177/070674379203700209

67. Agapejev S. [Clinical and epidemiological aspects of neurocysticercosis in Brazil: a critical approach]. Arq Neuropsiquiatr. 2003;61 (3B):822-828. doi:10.1590/s0004-282x2003000500022

68. Almeida SMD, Gurjão SA. Frequency of depression among patients with neurocysticercosis. Arq Neuropsiquiatr. 2010;68(1):76-80. doi:10.1590/s0004-282x2010000100017

69. Medina-Ortega Á, López-Valencia D, Vásquez-Arteaga LR. Recurrent neurocysticercosis of the frontal lobe. Case Reports. 2018;4:46-53. doi:10.15446/cr.v4n1.65860

70. Bhattarai R, Budke CM, Carabin H, et al. Quality of life in patients with neurocysticercosis in Mexico. Am J Trop Med Hyg. 2011;84 (5):782-786. doi:10.4269/ajtmh.2011.10-0646

71. Capitão CG. Changes in personality caused by neurocysticercosis. Psychology. 2016;07(01):92-100. doi:10.4236/psych.2016.71011

72. Garcia HH, Del Brutto OH. Taenia solium cysticercosis. Infect Dis Clin North Am. 2000;14(1):97-119, ix. doi:10.1016/s0891-5520(05) 70220-8

73. Garcia HH, Del Brutto OH. Neurocysticercosis: updated concepts about an old disease. Lancet Neurol. 2005;4(10):653-661. doi:10.1016/S1474-4422(05)70194-0 
74. Brotto W. Aspectos neurolÃ $\mid$ textthreesuperiorgicos da cisticercose. Arq Neuropsiquiatr. 1947;5:258-294. doi:10.1590/S0004282X1947000300005

75. Forlenza OV, Filho AH, Nobrega JP, et al. Psychiatric manifestations of neurocysticercosis: a study of 38 patients from a neurology clinic in Brazil. J Neurol Neurosurg Psychiatry. 1997;62(6):612-616. doi:10.1136/jnnp.62.6.612

76. Levav M, Mirsky AF, Cruz ME, Cruz I. Neurocysticercosis and performance on neuropsychologic tests: a family study in Ecuador. Am J Trop Med Hyg. 1995;53(5):552-557. doi:10.42 69/ajtmh.1995.53.552

77. Rodrigues CL, de Andrade DC, Livramento JA, et al. Spectrum of cognitive impairment in neurocysticercosis: differences according to disease phase. Neurology. 2012;78(12):861-866. doi:10. 1212/WNL.0b013e31824c46d1

78. Del Brutto OH, Nash TE, White ACJ, et al. Revised diagnostic criteria for neurocysticercosis. J Neurol Sci. 2017;372:202-210. doi:10.1016/j.jns.2016.11.045

79. Sharma BS, Banerjee AK, Kak VK. Intramedullary spinal cysticercosis. Case report and review of literature. Clin Neurol Neurosurg. 1987;89(2):111-116. doi:10.1016/0303-8467(87) 90185-5

80. Lezak MD, Howieson DB, Loring DW, Hannay HJ, Fischer JS. Neuropsychological Assessment. 4th ed. Oxford University Press; 2004.

81. McCormick GF. Cysticercosis-review of 230 patients. Bull Clin Neurosci. 1985;50:76-101.

82. Srivastava S, Chadda RK, Bala K, Majumdar P. A study of neuropsychiatric manifestations in patients of neurocysticercosis. Indian J Psychiatry. 2013;55(3):264-267. doi:10.4103/0019-5545.117146

83. Loftis JM, Huckans M, Ruimy S, Hinrichs DJ, Hauser P. Depressive symptoms in patients with chronic hepatitis $\mathrm{C}$ are correlated with elevated plasma levels of interleukin-1beta and tumor necrosis factor-alpha. Neurosci Lett. 2008;430(3):264-268. doi:10.1016/j. neulet.2007.11.001

84. Biswas A, Prasad AAK. Cysticercal dementia. J Assoc Physicians India. 1998;46:569.

85. Machado-Porto GCL, Lucato LT, Porto FHDG, de Souza EC, Nitrini R. Demência reversível por neurocisticercose: melhora da forma racemosa com o uso de anti-histamínicos. Dement Neuropsychol. 2015;9(1):85-90. doi:10.1590/S1980-57642015 DN91000014

86. Shah R, Chakrabarti S. Neuropsychiatric manifestations and treatment of disseminated neurocysticercosis: a compilation of three cases. Asian J Psychiatr. 2013;6(4):344-346. doi:10.1016/j.ajp.2013.01.013
87. Torrealba G, Del Villar S, Tagle P, Arriagada P, Kase CS. Cysticercosis of the central nervous system: clinical and therapeutic considerations. J Neurol Neurosurg Psychiatry. 1984;47(8):784-790. doi:10.1136/jnnp.47.8.784

88. Mahajan SK, Machhan PC, Sood BR, et al. Neurocysticercosis presenting with psychosis. J Assoc Physicians India. 2004;52:663-665.

89. Mustafa N, Al Ayyat D, Awad M, Elamin M, Bin Haider E, Alkhoori S. Neuropsychiatric manifestations of neurocysticercosis. Dubai Med J. 2020;3(1):37-40. doi:10.1159/000506365

90. García HH, Del Brutto $\mathrm{OH}$. Imaging findings in neurocysticercosis. Acta Trop. 2003;87(1):71-78. doi:10.1016/s0001-706x(03)00057-3

91. George U, Bansal G, Rathore S. MR spectroscopy aids diagnosis in subarachnoid space cysticercosis. Neurol India. 2010;58(6):957-958. doi: $10.4103 / 0028-3886.73763$

92. Handali S, Gonzalez AE, Hancock K, et al. Porcine antibody responses to taenia solium antigens rGp50 and sTs18var1. Am J Trop Med Hyg. 2004;71(3):322-326. doi:10.4269/ajtmh.2004.71.322

93. Handali S, Klarman M, Gaspard AN, et al. Development and evaluation of a magnetic immunochromatographic test to detect Taenia solium, which causes taeniasis and neurocysticercosis in humans. Clin Vaccine Immunol. 2010;17(4):631-637. doi:10.11 28/CVI.00511-09

94. Handali S, Klarman M, Gaspard AN, et al. Multiantigen print immunoassay for comparison of diagnostic antigens for Taenia solium cysticercosis and taeniasis. Clin Vaccine Immunol. 2010;17 (1):68-72. doi:10.1128/CVI.00339-09

95. Scheel CM, Khan A, Hancock K, et al. Serodiagnosis of neurocysticercosis using synthetic $8-\mathrm{kD}$ proteins: comparison of assay formats. Am J Trop Med Hyg. 2005;73(4):771-776. doi:10.4269/ ajtmh.2005.73.771

96. Wilson M, Bryan RT, Fried JA, et al. Clinical evaluation of the cysticercosis enzyme-linked immunoelectrotransfer blot in patients with neurocysticercosis. J Infect Dis. 1991;164(5):1007-1009. doi:10.1093/infdis/164.5.1007

97. Rodriguez S, Wilkins P, Dorny P. Immunological and molecular diagnosis of cysticercosis. Pathog Glob Health. 2012;106 (5):286-298. doi:10.1179/2047773212Y.0000000048

98. Tsang VC, Brand JA, Boyer AE. An enzyme-linked immunoelectrotransfer blot assay and glycoprotein antigens for diagnosing human cysticercosis (Taenia solium). J Infect Dis. 1989;159(1):50-59. doi:10.1093/infdis/159.1.50

99. Bouteille B. Epidemiology of cysticercosis and neurocysticercosis. Med Sante Trop. 2014;24. doi:10.1684/mst.2014.0378
Neuropsychiatric Disease and Treatment

\section{Publish your work in this journal}

Neuropsychiatric Disease and Treatment is an international, peerreviewed journal of clinical therapeutics and pharmacology focusing on concise rapid reporting of clinical or pre-clinical studies on a range of neuropsychiatric and neurological disorders. This journal is indexed on PubMed Central, the 'PsycINFO' database and CAS, and is the official journal of The International Neuropsychiatric Association (INA). The manuscript management system is completely online and includes a very quick and fair peer-review system, which is all easy to use. Visit http://www.dovepress.com/testimonials.php to read real quotes from published authors. 